\title{
Using Recurrence Plots to Visualize the Temporal Dynamics of Tutor/Student Interactions
}

\author{
Samuel A. Brian, Linda P. Salamanca, Jacob Whitehill, Judy S. Reilly, Marian S. Bartlett, Daniel \\ Angus, and Janet Wiles, Member, IEEE
}

\begin{abstract}
Non-verbal behavior is a mode of human interaction that is critical to communication, especially in situations where speech is sparse. In this study, we developed novel visualizations (called behavioral recurrence plots) to reveal the non-verbal behavior patterns of a tutor instructing a thirteen year old student in a mathematical problem, and the time-locked responses of the student solving a series of problems. In the first phase of analysis, video recordings were manually annotated to document the participants' speech, behavior, gesturing, fidgeting, and eye gaze over time, and automatic detection of movement was used to extract gross human motion. In a second phase of analysis, recurrence plots were used to visualize the interaction dynamics between the tutor's behaviors and between the tutor and student. The recurrence plots reveal the global structure of the tutoring session, points of interest and transitions, as well as interactions between the many modes of non-verbal communication.
\end{abstract}

Index Terms-human interaction, human motion, learning dynamics, recurrence plots

\section{INTRODUCTION}

$\mathrm{T}$ he many modes of human interaction are critical to communication. Observing the behavioral relationship between humans can give an insight into conscious and unconscious aspects of interaction and their temporal relations over a range of time scales. Non-verbal behaviors, such as body language and movement, convey critical information, especially in situations where speech is sparse.

Information visualization is a useful method for revealing interaction dynamics at multiple scales, from sub-second to tens of minutes. Time series analysis techniques are increasingly being recruited for use in social sciences. One such technique, called recurrence plotting $[1,2]$, can be used to visualize the temporal structure within and between coupled time series. One main advantage of recurrence plot

Manuscript received June 29, 2012. This work was supported by National Science Foundation Collaborative grant \#IIS-0905661, 'Computational analysis of nonverbal behavior in adaptive tutoring' and the NSF Temporal Dynamics of Learning Center.

S. Brian, D. Angus and J Wiles are with the University of Queensland, Brisbane, Australia (corresponding author J Wiles phone +61-7-3365-2902, fax+61-7-3365-4999, email: j.wiles@uq.edu.au).

L. Salamanca and J. Reilly are with San Diego State University.

J. Whitehill and M. Bartlett are with the University of California, San Diego. visualization is the ability to view an entire time series at a glance, to select times at which transitions or other interesting events occur, and to identify regions for detailed analysis.

The analyses in this paper are of a video recorded tutorial session which was collected as part of a collaborative study between the Machine Perception Lab at UCSD and the Developmental Laboratory for Language and Cognition at SDSU. A thirteen year old student participated in a 10-minute pretest on logarithms, followed by a 40-minute one-on-one teaching session focused on logarithms, and then a 10-minute posttest. All speech and non-verbal behaviors (e.g., gestures and eye gaze) were manually annotated into ELAN [3].

The non-verbal behavior annotations were visualized with recurrence plots to emphasize interaction and recurrent trends. Visual motion signals were extracted from both the student and tutor videos to explore human motion on a sub-second time scale. The method used was to apply the mean-absolutedifference metric [4] to the disparity in luma channel images applied to each pair of consecutive video frames in both videos to generate two time series signals which represent the motion of each participant.

\section{RESUlTS AND DiSCUSSION}

A recurrence plot of the tutor's gestures and behavior over a short segment of the session is shown in Fig. 1. Bar graphs of student and tutor fidgeting annotations were plotted in Fig. 2. Eye gaze annotations of both participants were visualized with a recurrence plot in Fig. 3.

The motion signals extracted from the tutor and student videos were imported into ELAN where they were checked for consistency with non-verbal annotations (see Fig. 4). A color coded recurrence plot of the motion signals is shown in Fig. 5.

The visual motion signal extracted from video was found to be responsive to gestures, fidgeting, and other large movements, which could aid the manual annotation process by visually suggesting sections of significant motion and reduce the amount of time required to process whole videos.

This type of interaction analysis contributes to understanding the dynamics of student-teacher interactions: 1) it augments our understanding of the non-verbal structure in communication; 2) it reveals the temporal dynamics of the interaction; and 3) practically, it can to serve to enhance teaching strategies. 


\section{ACKNOWLEDGMENT}

Support for this work was provided by NSF grants IIS0905622, IIS-0905661, and NSF REU.

\section{REFERENCES}

D. Angus, A. Smith, and J. Wiles, "Conceptual Recurrence Plots: Revealing Patterns in Human Discourse," IEEE Transactions on Visualization and Computer Graphics, vol. PP, pp. 1-1, 2012. J.-P. Eckmann, S. O. Kamphorst, and D. Ruelle, "Recurrence Plots of Dynamical Systems," Europhysics Letters, vol. 4, pp. 973-977, 1987.

L. A. Technology. (15/03). ELAN - Language Archiving

Technology. Available: http://www.lat-mpi.eu/tools/elan/

S. R. Subramanya, H. Patel, and I. Ersoy, "Performance evaluation of block-based motion estimation algorithms and distortion measures," in Proceedings of the International Conference on Information Technology: Coding and Computing, 2004, pp. 2-7 Vol.2.

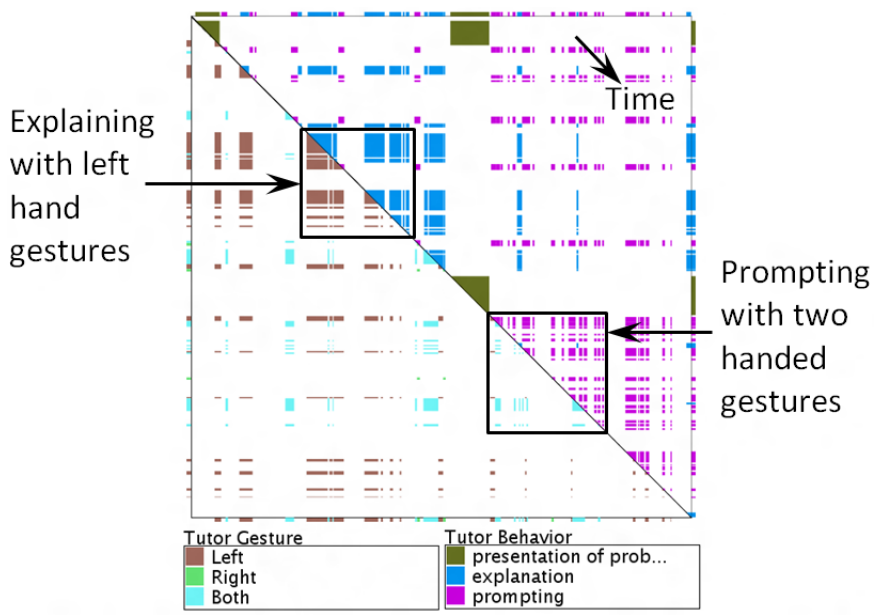

Fig. 1. Recurrence plot of tutor hand gesture and tutor behavior annotations between times 30:25.0 and 37:11.6. The right hand and bi-manual gestures in this section are associated with prompting. The left hand gestures are predominately associated with the explanation behavior, which is feasible as they are both the most common events in their respective classes of annotations. Although these trends are not strictly adhered to, they are generally followed throughout the tutorial session.

\section{Fidgeting Occurrences}

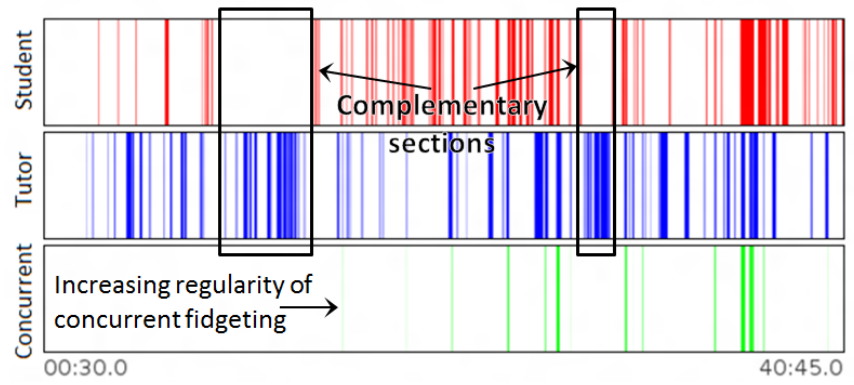

Fig. 2. Student (red), tutor (blue), and concurrent (green) fidget annotations between times 00:30.0 and 40:45.0. The areas highlighted in the figure show extended periods where the student does not fidget while the tutor has episodes of regular fidgeting. Concurrent fidgeting rarely occurs at the beginning of the session, but simultaneous actions occur more often and for longer durations as the session progresses.

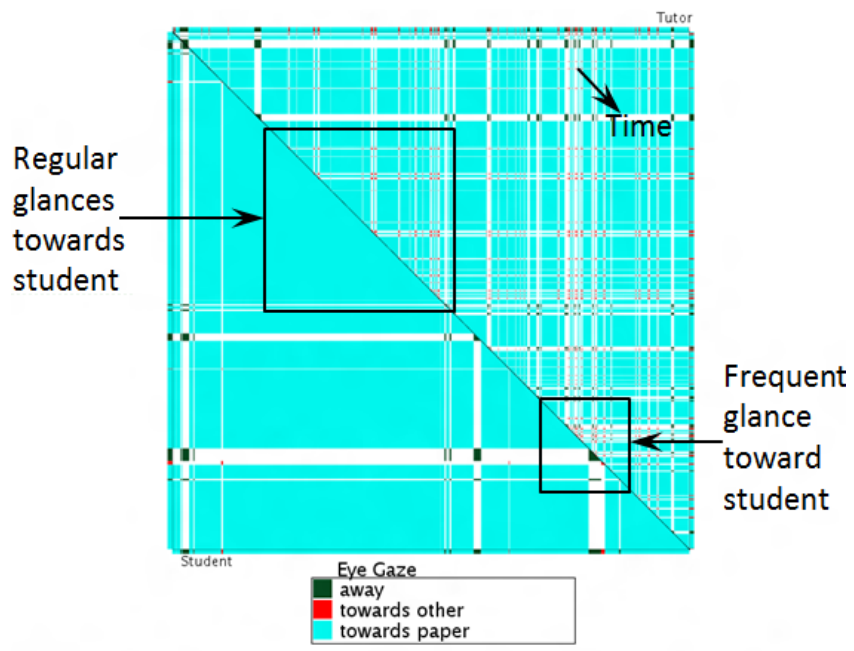

Fig. 3. Recurrence plots of tutor and student eye gaze annotations between times 30:25.0 and 37:11.6. The student spends the most time looking at the paper in front of him as he attempts a problem, and occasionally glances away to the rest of the room or the tutor. The tutor also watches the page, but regularly glances towards the student whilst explaining. There are episodes of very frequent glances from the tutor towards the student which correspond to the tutor's prompting.

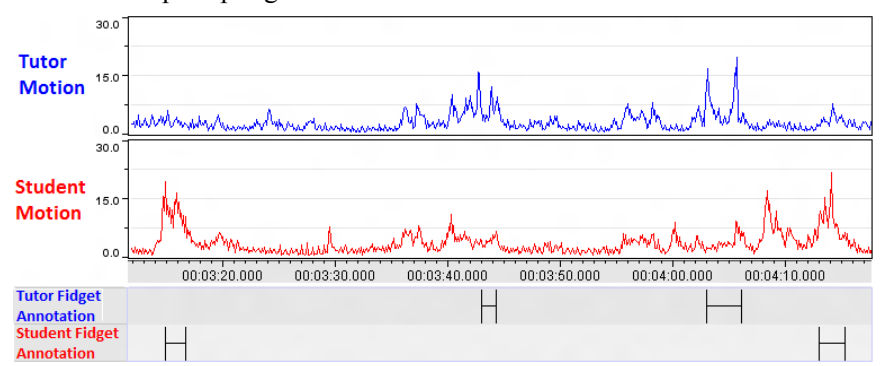

Fig. 4. Tutor and student motion signals and corresponding fidget annotations between times 3:10 and 4:20. Both the student and tutor fidget events relate to definite activity in the respective motion signals. Pairs of peaks recur throughout the video and are caused by such events as a subject moving their hand to their face, pausing briefly, and returning their hand to its resting position.

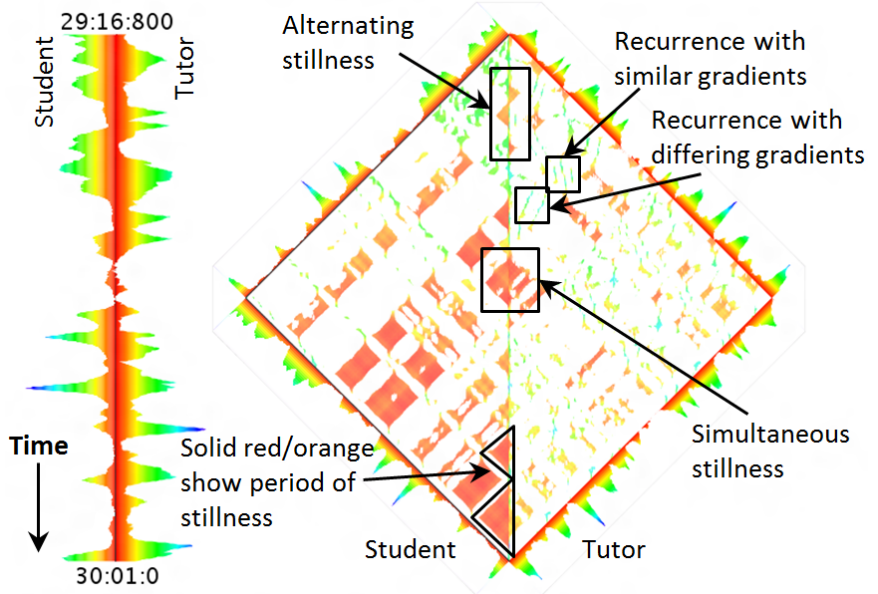

Fig. 5. Diagonally adjacent signal value color coded recurrence plots of student (left) and tutor (right) motion signals from time 29:16.5 to 30:01.3 with a recurrence threshold of 0.1 and a comparison vector including points 3 before/after. It is apparent that the student's motion signal has sections of consistent and low motion, corresponding to periods of stillness. There are occurrences where the student and tutor are still at the same time, but there are also occasions where they alternate complementary stillness. 